\title{
Erratum to: Preservation of Genital Innervation in Women During Total Mesorectal Excision: Which Anterior Plane?
}

\author{
Frédérique Peschaud • David Moszkowicz • \\ Bayan Alsaid · Thomas Bessede • Christophe Penna • \\ Gérard Benoit
}

Published online: 9 December 2011

(C) Société Internationale de Chirurgie 2011

\section{Erratum to: World J Surg \\ DOI 10.1007/s00268-011-1313-2}

In the original article the captions for Figs. 4 and 5 are transposed. Following are the figures with their correct corresponding captions:

The online version of the original article can be found under doi:10.1007/s00268-011-1313-2.

F. Peschaud - D. Moszkowicz · B. Alsaid - T. Bessede .

G. Benoit

Laboratory of Experimental Surgery, EA 4122, Faculty of

Medicine, Paris Sud-11 University, 78, rue du Général Leclerc,

94275 Le Kremlin-Bicêtre, France

F. Peschaud - C. Penna

Department of Surgery Assistance Publique, Hôpitaux de Paris,

Ambroise Paré Hospital, 9 avenue Charles-de-Gaulle,

92 Boulogne-Billancourt, France

F. Peschaud · C. Penna

University of Versailles Sain-Quentin-en-Yvelines (UVSQ),

55 avenue de Paris, 78035 Versailles, France

\section{F. Peschaud ( $\square)$}

Service de Chirurgie Oncologique et Digestive, Hôpital

Ambroise Paré, 9 Avenue Charles de Gaulle,

92100 Boulogne, France

e-mail: frederique.peschaud@apr.aphp.fr

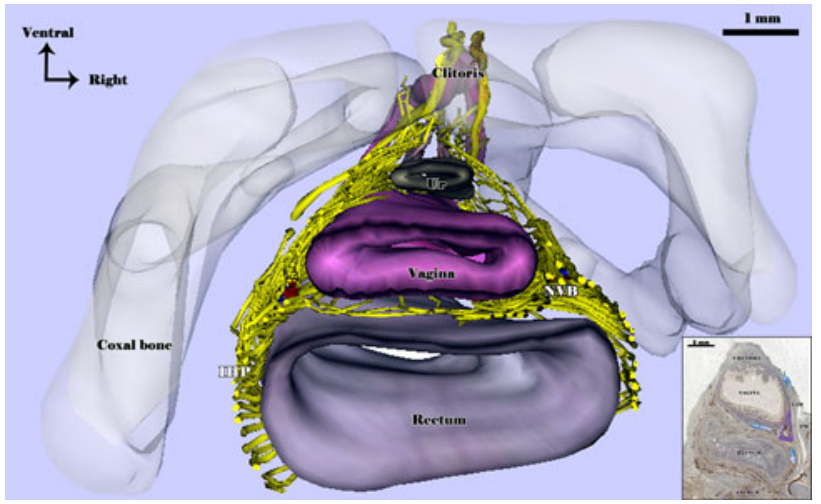

Fig. 4 Three-dimensional (3D) view of fetus intrapelvic organs with pelvic nerves. Superior infraperitoneal 3D view of a 30 -week-old female fetus: intrapelvic organs with immunolabeled pelvic nerves showing the position of the inferior hypogastric plexus (IHP) on the lateral aspects of the rectum. From the IHP, efferent branches rise in distal directions: postero-inferior for the rectal wall, lateral for the levator ani muscles, and antero-inferior to form the NVBs. Some fibers from the NVBs branch medially to innervate the posterior vaginal wall. More caudally, the bundle puts out fibers in three major projections: an anterior projection for the urethral sphincter complex, an anterolateral projection (the cavernous nerve) that travels anterolaterally to the vagina to reach the corpora cavernosa, and a posterolateral projection (the spongious nerve) that continues posterolaterally to the vagina to innervate the corpus spongiosum. In inset, blue arrows show the directions of the IHP and NVB main terminal efferences. $U r$ urethra 


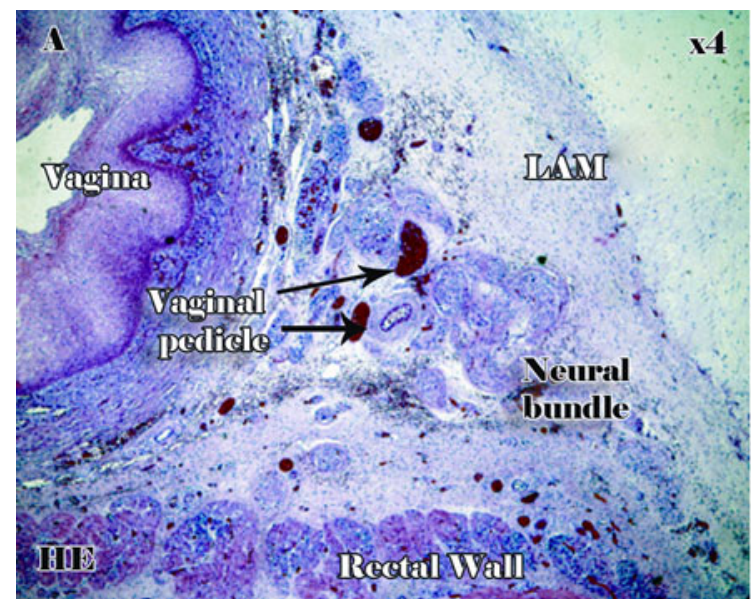

Fig. 5 Morphologic and functional systematization of the NVB. Histologic 5 - $\mu$ m-thick transverse sections of a 30 -week-old female fetus scanned at an optical resolution of 4,800 dpi, stained with hematoxylin/eosin (a) and immunolabeled with anti-S100 antibody (b), anti-TH (c), anti-nNOS (d), and anti-VAChT (e). Neurovascular
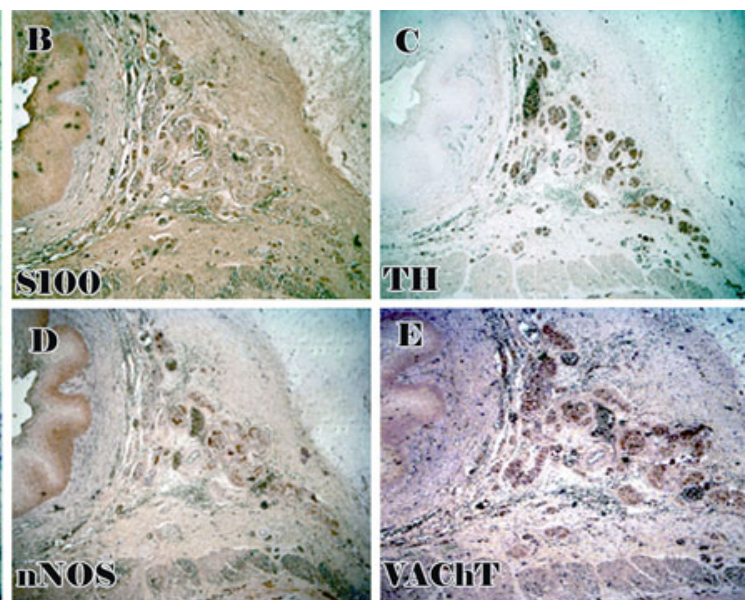

bundles consist of nerve fibers and vaginal blood vessels scattered throughout the posterior and anterior extension of the RVS in a triangularly shaped space. $L A M$ levator ani muscle; $P M$ piriform muscle; $P N$ pudendal nerve 